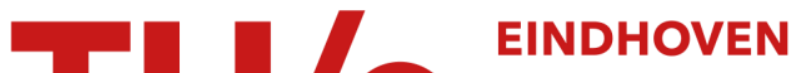 UNIVERSITY OF TECHNOLOGY
}

\section{Nucleation at high pressure II: Wave tube data and analysis.}

Citation for published version (APA):

Luijten, C. C. M., \& Dongen, van, M. E. H. (1999). Nucleation at high pressure II: Wave tube data and analysis. Journal of Chemical Physics, 111(18), 8535-8544. https://doi.org/10.1063/1.480194

DOI:

10.1063/1.480194

Document status and date:

Published: 01/01/1999

\section{Document Version:}

Publisher's PDF, also known as Version of Record (includes final page, issue and volume numbers)

\section{Please check the document version of this publication:}

- A submitted manuscript is the version of the article upon submission and before peer-review. There can be important differences between the submitted version and the official published version of record. People interested in the research are advised to contact the author for the final version of the publication, or visit the $\mathrm{DOI}$ to the publisher's website.

- The final author version and the galley proof are versions of the publication after peer review.

- The final published version features the final layout of the paper including the volume, issue and page numbers.

Link to publication

\section{General rights}

Copyright and moral rights for the publications made accessible in the public portal are retained by the authors and/or other copyright owners and it is a condition of accessing publications that users recognise and abide by the legal requirements associated with these rights.

- Users may download and print one copy of any publication from the public portal for the purpose of private study or research.

- You may not further distribute the material or use it for any profit-making activity or commercial gain

- You may freely distribute the URL identifying the publication in the public portal.

If the publication is distributed under the terms of Article 25fa of the Dutch Copyright Act, indicated by the "Taverne" license above, please follow below link for the End User Agreement:

www.tue.nl/taverne

Take down policy

If you believe that this document breaches copyright please contact us at:

openaccess@tue.nl

providing details and we will investigate your claim. 


\title{
Nucleation at high pressure. II. Wave tube data and analysis
}

\author{
C. C. M. Luijten, P. Peeters, and M. E. H. van Dongen ${ }^{\text {a) }}$ \\ Eindhoven University of Technology, Department of Applied Physics, P.O. Box 513, 5600 MB Eindhoven, \\ The Netherlands
}

(Received 3 March 1999; accepted 10 August 1999)

\begin{abstract}
Nucleation rate data, obtained from expansion wave tube experiments, are reported for several vapor-gas mixtures at high pressure. Results are given for water-vapor in the presence of helium and nitrogen gas, and for $n$-nonane in helium and methane. For all these mixtures, carrier gas pressures of 10, 25, and 40 bar have been applied, with temperatures ranging from 230 to $250 \mathrm{~K}$. An extended form of the nucleation theorem (in terms of the derivative of the nucleation rate with respect to carrier gas pressure) is derived, which appears to be very helpful in the interpretation of high pressure data. It can be used to obtain the carrier gas content of the critical nucleus directly from the pressure dependence of experimental nucleation rates. Combining this method with the theoretical considerations of part I of this paper [J. Chem. Phys. 111, 8524 (1999), preceding paper]: the nucleation behavior of water at high pressures of both helium and nitrogen can quantitatively be understood. For $n$-nonane in helium our "pressure perturbation approach" is also valid. For $n$-nonane in methane, however, this approach fails because of the high methane solubility in the liquid phase. (C) 1999 American Institute of Physics. [S0021-9606(99)51841-2]
\end{abstract}

\section{INTRODUCTION}

Although today's literature on nucleation rate measurements is extensive, the available amount of high pressure data is quite surveyable. Yet, high pressure measurements can reveal important information, mainly in two respects. First of all, there are many practical circumstances where nucleation takes place in the presence of a high pressure carrier gas. Chemical plants and natural gas transport lines are among the most important examples where high pressure nucleation may take place. Second, nucleation experimentalists have nearly always added carrier gases to their nucleating vapors. For the question of a possible carrier influence to be resolved, it is necessary to measure at elevated carrier gas pressures.

Total pressures in quantitative nucleation experiments have usually not exceeded a few bars thus far. ${ }^{1}$ Moreover, results are sometimes conflicting: ${ }^{2}$ Users of expansion cloud chambers (ECC) usually report null carrier gas influence, whereas in thermal diffusion cloud chambers (TDCC) severe pressure effects are reported already at relatively low pressures. ${ }^{3}$

Only few studies exist that go up to several tens of bars. Heist et $a l^{4}{ }^{4}$ reported a strong increase of the critical supersaturation of $n$-propanol with pressure for helium and hydrogen, implying a dramatic decrease of nucleation rate with pressure. Kane and El-Shall, ${ }^{5}$ also using a TDCC, confirmed this observation for the glycerol-helium system. It has recently become clear, however, that the range of conditions for reliable TDCC operation itself depends on total pressure. $^{6-8}$

Muitjens ${ }^{9}$ applied an ECC to investigate several vaporgas systems. For water-helium no pressure trend was found

${ }^{a)}$ Electronic mail: m.e.h.v.dongen@phys.tue.nl up to 57 bar. In water-nitrogen, the nucleation rate was found to increase by two orders of magnitude for a pressure of 60 bar. Luijten et al. ${ }^{10}$ found the same behavior, using the expansion wave tube setup that is also applied in this work. With the same setup, Looijmans et al. ${ }^{11}$ studied the nucleation behavior of $n$-nonane in methane. They observed severe pressure effects, but these were partially attributed to the carrier gas being dissolved into the nucleating drops, which does not generally occur for supercritical gases.

In Sec. IV of this paper we present a comprehensive series of high pressure measurements. Nucleation isotherms of water are reported in carrier gases of helium and nitrogen, at pressures of 10, 25, and 40 bar. For $n$-nonane, nucleation isotherms were measured in helium and methane for the same pressure conditions, with temperatures ranging from 230 to $250 \mathrm{~K}$. The above choice of systems is based on the idea of varying the degree of gas-vapor interaction. Each nucleating vapor is investigated in an environment of a noninteracting carrier gas (helium), in combination with a carrier that interacts weakly (water-nitrogen) or strongly (n-nonane-methane) with the vapor.

All data were measured using the pulse expansion wave tube developed by Looijmans et al. ${ }^{12,13}$ However, we have improved both the preparation procedure and the composition measurement of the gas-vapor mixtures. To that end, the setup was extended with a newly built mixture preparation unit, see Sec. III. Two methods for vapor fraction measurement were developed (applicable to water and hydrocarbons, respectively). For the calibration of these methods, the same mixture preparation unit was used.

In the analysis of the new data, we compare the measured nucleation rates with results of the theoretical models that were extended for high pressure use in part I of this paper (hereafter referred to as Paper I). ${ }^{14}$ However, important information can also be abstracted from the measurements 
themselves, without reference to a particular nucleation model. In Sec. II, we present an analysis based on the wellknown nucleation theorem. By rewriting the two nucleation theorems for a binary mixture as two independent derivatives of the nucleation rate- one with respect to supersaturation, the other with respect to pressure-it is possible to obtain both the vapor and carrier gas content of the critical cluster.

\section{NUCLEATION THEOREMS AND TOTAL PRESSURE}

For the classical nucleation model, a relation between the critical work of formation and the supersaturation is easily derived. Kashchiev in 1982 proved the validity of this so-called nucleation theorem without invoking the classical (capillarity) model. ${ }^{15}$ Later it was extended to mixtures-and proven to hold without any assumptions about the shape, size, and composition of the critical nucleus-by Oxtoby and Kashchiev. ${ }^{16}$ In their original notation it reads (for an isothermal system)

$$
\frac{\partial W^{*}}{\partial \mu_{0, i}}=-\Delta n_{i}^{*},
$$

where $W^{*}$ denotes the work of formation of a critical cluster and $\mu_{0, i}$ is the chemical potential of component $i$ in the gaseous phase. The quantity $\Delta n_{i}^{*}$ denotes the excess number of molecules of component $i$ in the critical cluster, over that in the same volume of the gas phase, so $\Delta n_{i}^{*}=n_{i}^{*}-n_{i, 0}$.

Recently, a second nucleation theorem was proven by Ford, which relates the temperature dependence of the nucleation rate (at fixed supersaturation) to the excess internal energy of the critical cluster. ${ }^{17,18}$ Yet, in the present work we consider isothermal nucleation, and therefore, use Eq. (1) only. In view of our interest in carrier gas effects, we will rewrite this expression in terms of the derivative of the nucleation rate with respect to total pressure $p$.

We follow the approach of Oxtoby and Laaksonen, ${ }^{19}$ who consider the vapor-gas mixture as a normal binary system. They then apply the nucleation theorem for both components, leading to relations between experimentally observable derivatives of the nucleation rate and the excess numbers $\Delta n_{i}^{*}$ with respect to supersaturation and total pressure.

The above authors then estimate these excess numbers using the virial equation of state, and conclude that their estimates do not agree with existing diffusion cloud chamber data of Heist et al. ${ }^{4}$ and Katz et al. ${ }^{20}$ We will demonstrate below how the numbers of vapor and gas molecules in the critical cluster (denoted as $n_{\vee}^{*}$ and $n_{g}^{*}$, respectively) can be obtained from experimental data, without reference to an equation of state.

Expressing the nucleation rate in its most general form as

$$
J=K \exp \left(-W^{*} / k_{B} T\right),
$$

two general relations can be derived with the help of the nucleation theorem (1) and the chain rule

$k_{B} T\left[\frac{\partial \ln (J / K)}{\partial S}\right]_{p, T}=\Delta n_{\vee}^{*}\left(\frac{\partial \mu_{\mathrm{v}}}{\partial S}\right)_{p, T}+\Delta n_{g}^{*}\left(\frac{\partial \mu_{g}}{\partial S}\right)_{p, T}$,
$k_{B} T\left[\frac{\partial \ln (J / K)}{\partial p}\right]_{S, T}=\Delta n_{\mathrm{v}}^{*}\left(\frac{\partial \mu_{\mathrm{v}}}{\partial p}\right)_{S, T}+\Delta n_{g}^{*}\left(\frac{\partial \mu_{g}}{\partial p}\right)_{S, T}$.

The derivatives of $\mu_{g}$ are easily evaluated, assuming that $\mu_{g}$ is not significantly affected by the (very small) vapor fraction. In that case, the last term of Eq. (3) vanishes. The pressure derivative follows from the Gibbs-Duhem relation for the pure gas:

$$
\left(\frac{\partial \mu_{g}}{\partial p}\right)_{S, T}=\mathrm{v}_{g}=\frac{Z_{g} k_{B} T}{p}
$$

where $\mathrm{v}_{g}$ and $Z_{g}$ are the molecular volume and compressibility factor of the gas. From the definition of supersaturation, $S=\exp \left(\Delta \mu / k_{B} T\right)$, we have for the vapor

$$
\left(\frac{\partial \mu_{\mathrm{v}}}{\partial S}\right)_{p, T}=\frac{k_{B} T}{S} .
$$

Again referring to the definition of $S$, the pressure derivative of $\mu_{\mathrm{v}}$ at constant $S$ equals the pressure derivative of $\mu_{\mathrm{v}}^{\mathrm{eq}}$. The latter quantity is expressed by

$$
\mu_{\mathrm{v}}^{\mathrm{eq}}=\mu_{\mathrm{v}}^{\bigcirc}+\ln \left(\frac{\phi_{\mathrm{v}}^{\mathrm{eq}} y_{\mathrm{v}}^{\mathrm{eq}} p}{\mathcal{F}^{\bigcirc}}\right),
$$

where $\mathcal{F}^{\bigcirc}$ is the reference fugacity. Substituting Eq. (1) of Paper I for the equilibrium vapor fraction, and assuming the liquid mixture to be ideal, the pressure derivative reads

$$
\left(\frac{\partial \mu_{\mathrm{v}}}{\partial p}\right)_{S, T}=k_{B} T\left[\frac{1}{x_{\mathrm{v}}^{\mathrm{eq}}} \frac{\partial x_{\mathrm{v}}^{\mathrm{eq}}}{\partial p}+\frac{\mathrm{v}^{l}}{k_{B} T}\right],
$$

where $x_{\mathrm{v}}^{\mathrm{eq}}$ is the equilibrium molar fraction of vapor molecules in the liquid phase. With the help of Henry's law, the first term in brackets can be approximated as $-x_{g}^{\mathrm{eq}} / p$, where $x_{g}^{\mathrm{eq}}$ is the fraction of carrier gas in the liquid phase at equilibrium.

For the derivatives of the pre-exponential factor $K$ in Eqs. (3) and (4), one has to rely on a kinetic model. This hardly causes loss of generality, since the proportionality of the prefactor to both $\rho_{1}$ and $\rho_{1}^{\mathrm{eq}}$ is a general feature of kinetic nucleation models. ${ }^{16}$ Neglecting a possible pressure dependence of surface tension in the prefactor, we have $K$ $\propto S\left(f_{e} / Z_{g}\right)^{2}$-see Eq. (12) of Paper I-where both the enhancement factor $f_{e}$ and the gas compressibility factor $Z_{g}$ are functions of pressure and temperature. Obviously, the derivative with respect to $S$ is

$$
\left(\frac{\partial \ln K}{\partial \ln S}\right)_{p, T}=1
$$

Assuming $f_{e}$ to have the form $\exp \left[\beta\left(p-p^{s}\right)\right] \approx e^{\beta p}$, which is reasonable in most cases, the pressure dependence of $K$ is given by

$$
\left(\frac{\partial \ln K}{\partial \ln p}\right)_{S, T}=2 \ln f_{e}-2\left(\frac{\partial \ln Z_{g}}{\partial \ln p}\right)_{T} .
$$

The derivative on the right hand side can be estimated from a generalized compressibility chart in terms of reduced pressure and temperature (see, for example, Ref. 21). For all nucleation conditions presented in Sec. IV, it appears to be 
much smaller than unity. Yet, the first term on the right hand side can be appreciable when the enhancement factor significantly exceeds unity.

With help of the above considerations, the general expressions (3) and (4) transform into

$$
\begin{aligned}
& {\left[\frac{\partial \ln J}{\partial \ln S}\right]_{p, T}=\Delta n_{\mathrm{v}}^{*}+1} \\
& {\left[\frac{\partial \ln J}{\partial \ln p}\right]_{S, T}=\Delta n_{\mathrm{v}}^{*}\left(\frac{p \mathrm{v}^{l}}{k_{B} T}-x_{g}^{\mathrm{eq}}\right)+\Delta n_{g}^{*} Z_{g}+2 \ln f_{e} .}
\end{aligned}
$$

Finally, when replacing the excess numbers $\Delta n_{i}^{*}$ by the real numbers of molecules $n_{i}^{*}$ in the critical cluster, we can arrive at a clear interpretation of these relations. It is easily proven that $n_{\mathrm{v}, 0}$, being the number of vapor molecules present in one critical cluster volume of the gas phase, is negligible for the dilute vapors under study: $\Delta n_{\mathrm{v}}^{*}=n_{\mathrm{v}}^{*}$. Since the critical cluster consists mainly of vapor, its volume can be approximated as $n_{\mathrm{v}}^{*} \mathrm{v}^{l}$. The same volume in the gas phase contains $n_{g, 0}=n_{\mathrm{v}}^{*} \mathrm{v}^{l} p /\left(Z_{g} k_{B} T\right)$ molecules. This leads to a cancellation of the first term between brackets in Eq. (12) with $n_{g, 0}$, and we obtain

$$
\begin{aligned}
& {\left[\frac{\partial \ln J}{\partial \ln S}\right]_{p, T}=n_{\mathrm{v}}^{*}+1,} \\
& {\left[\frac{\partial \ln J}{\partial \ln p}\right]_{S, T}=-n_{\mathrm{v}}^{*} x_{g}^{\mathrm{eq}}+n_{g}^{*} Z_{g}+2 \ln f_{e} .}
\end{aligned}
$$

Both left hand sides of the above equations are accessible to experiment. The first is simply the slope of a $(J, S)$-plot on a double logarithmic scale, whereas the second can be found from the distance between isotherms for different pressures in the same kind of graph. Equation (13) is the familiar single component result: The experimental slope of $J$ versus $S$ directly provides the number of vapor molecules involved in the critical cluster. The second relation will turn out to be very useful in the analysis of carrier gas effects further in this paper.

First consider the limiting case where the gas does not enter the cluster at all. Both $x_{g}^{\mathrm{eq}}$ and $n_{g}^{*}$ equal zero, so $\Delta n_{g}^{*}$ $=-n_{g, 0}$, meaning that the gas is simply expelled from the region where the critical cluster resides. Yet, the enhancement factor contribution indicates that the nucleation rate does increase with pressure, for kinetic reasons. Even if the thermodynamic driving force for nucleation-expressed by the supersaturation $S$-is kept constant, and the carrier gas is completely absent in the cluster, the nucleation rate increases simply because there are more vapor monomers present to do the job.

When the carrier gas enters the cluster, the situation gets more complicated. The first term on the right hand side of Eq. (14) represents the equilibrium dissolution of the carrier gas in the bulk liquid core of the cluster. Apparently, it causes the nucleation rate to decrease, which can be attributed to the decrease of the chemical potential of the vapor component in the cluster. Still, the carrier gas solubility is usually small enough for the first term to be neglected. The second term $\left(n_{g}^{*} Z_{g}\right)$ accounts for the total number of carrier
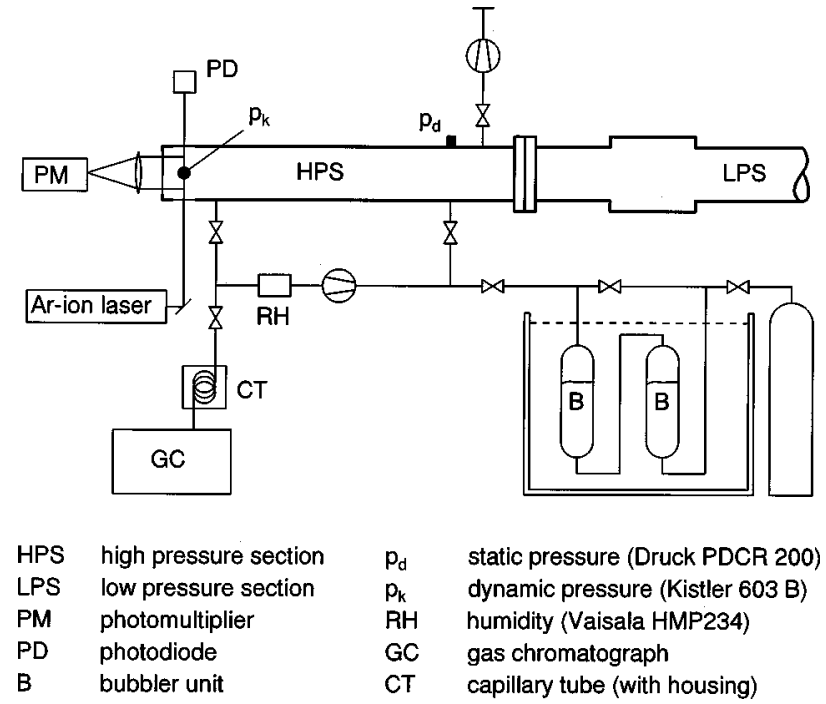

FIG. 1. Pulse-expansion wave tube setup for high pressure nucleation experiments.

gas molecules in the critical cluster, either dissolved in the bulk or adsorbed at the surface. When enhancement factors and equilibrium solubilities are known or estimated, $n_{g}^{*}$ can thus be derived from a measurement of the pressure dependence of $J$.

It is also possible to apply the relations (13) and (14) to the pressure perturbation model of Paper I, as is demonstrated in the Appendix. The consistency of the perturbation model with experimental data can therefore be interpreted in terms of critical cluster content.

\section{EXPANSION WAVE TUBE EXPERIMENTS}

The nucleation experiments were done in the pulse expansion wave tube setup developed by Looijmans et al. ${ }^{12,13}$ This set-up is based on the well-known nucleation pulse method, implemented in the driver section (high pressure section, HPS) of a shock tube. In order to create the pulse, the driven section (low pressure section, LPS) of the tube contains a relatively short segment having a slightly wider inner diameter. The set-up is sketched in Fig. 1, together with its peripherals that will be described below.

After preparation of the gas-vapor mixture, operation of the tube starts with rupture of the diaphragm between HPS and LPS. The initial expansion wave, traveling into the HPS, is followed in time by the reflections of the shock wave at the widening and the constriction, respectively. These reflections lead to the creation of a nucleation pulse at the end wall of the HPS. After the pulse, a nearly constant pressure level is maintained for a longer period of time, allowing the nucleated drops to grow to macroscopic sizes.

Constant Angle Mie Scattering under $90^{\circ}$ is applied to obtain the droplet radius as a function of time, using an argon-ion laser with a wavelength of $514.2 \mathrm{~nm}$. Simultaneously, the attenuation of the incident beam is measured, which is combined with the time-resolved radius in order to obtain the droplet concentration. ${ }^{12}$ The latter is divided by the duration of the pulse, yielding the nucleation rate $J$. 
The temperature during the experiment is calculated from the initial temperature and the dynamic pressure measurement, using the assumption of isentropicity and a real gas equation of state. ${ }^{10}$ Since the pressure during the pulse is not exactly constant, the nucleation pressure $p_{\text {exp }}$ is averaged over the pulse; the associated temperature $T_{\exp }$ is then assigned to the observed nucleation rate $J_{\exp }$. The possible influence of this averaging procedure on the results presented below is discussed elsewhere, ${ }^{21}$ and found to be very small.

In the ideal gas limit, it is only the pressure ratio between the expanded and initial states that matters. However, when real gas effects play a role, as they do for the nucleation conditions in our experiments, the nucleation temperature is sensitive to total pressure. Usually, this leads to an additional (Joule-Kelvin) cooling upon expansion. For nitrogen and methane, the extra cooling can amount up to several degrees for the conditions of interest here, and must be taken into account in order to derive meaningful temperatures (and, hence, supersaturations). Since the molar fraction of vapor is always very small at high pressure, its influence can be neglected in the temperature calculation.

Yet, the vapor fraction $y_{v}$ is the essential quantity that determines the supersaturation ratio $S$, see Eq. (3) of Paper I. Therefore, the highest possible accuracy is required to determine $y_{\mathrm{v}}$ in the high pressure mixture. Unfortunately, conventional methods can not be used. The vapor pressure method is not reliable here, because addition of the high pressure carrier gas will affect the wall adsorption equilibrium in the test section. Preparation of the mixture in a separate vessel, followed by flushing of the test section, would be impractical since huge amounts of gas are then required. In view of these problems, we have developed two independent methods for in situ determination of vapor fractions. One can be used for water-vapor, the other for hydrocarbons.

Humidity sensors exist for measuring the water content of gaseous mixtures, some of which can even be mounted in a high pressure environment. Still, we had been aware for some time that the device that we were using (Vaisala HMP 124B) showed an intrinsic deviation, depending on total pressure and the kind of carrier gas. Therefore, a calibration method was developed in cooperation with the manufacturer, based on controlled saturation of the carrier gas with water. ${ }^{23}$ The construction of the bubbler pair, also represented in Fig. 1 , was taken from a paper by Wylie and Fisher. ${ }^{24}$ The bubblers where then placed in a thermostatic bath, accurately controlling the saturation temperature.

Using the method of controlled saturation, we tested a newer sensor type (HMP 234), which was also used in the nucleation experiments described below. We compared the reading of the humidity output with the saturated value, taking into account the enhancement factor of water in the gases of interest. The pressure deviation in nitrogen gas turned out to be systematic, enabling one to recalculate the actual humidity from the indicated humidity and total pressure. Helium had no significant effect on the humidity signal.

For hydrocarbons, gas chromatography is a wellestablised method to measure vapor fractions. However, analysis of high pressure mixtures is again not straightforward. In order to prevent preliminary condensation of the mixture during pressure reduction on its way to the gas chromatograph (GC, type HP 5890II), a thermostatic capillary tube was mounted between the test section and the GC, see Fig. 1. The mixture enters the $\mathrm{GC}$ via a six-way rotator valve, which brings a reproducible amount of sample onto the GC column. After passage of the column, the mixture is analyzed in a flame ionization detector (FID), coupled to an automatic integrating device.

In order to calibrate the gas chromatographic setup, the same procedure was followed as in the calibration of the humidity sensor. A second bubbler pair was constructed and filled with $n$-nonane. At thermostatic conditions, helium was bubbled through the saturators; the saturated vapor content was calculated from a correlation for the saturated vapor pressure, ${ }^{25}$ corrected by the enhancement (Poynting) factor. By increasing the helium pressure in steps, the vapor fraction was varied in a range relevant to the nucleation experiments. The resulting calibration was double-checked using two cilinders containing certified calibration gases (BOC Speciality Gases, Surrey, UK).

The two-saturator calibration setup was also used as a mixture preparation unit in the nucleation experiment, see Fig. 1. After evacuation of both HPS and LPS to a pressure below $10 \mathrm{~Pa}$, dry carrier gas is led into the test section up to a certain pressure level $p_{\mathrm{dry}}$. Then the carrier gas is routed through the saturators, and the HPS is filled up to the desired initial pressure $p_{0}$.

In principle, the saturation temperature $T_{s}$ and the pressure levels $p_{\text {dry }}$ and $p_{0}$ fully determine the final vapor fraction. However, the actual vapor fraction always turned out to be lower than the predicted value (with several possible explanations, such as wall adsorption and the dead volume of the tubing between bubblers and HPS). Accounting for this difference - which reproduces reasonably-in advance, $p_{\text {dry }}$ could be adjusted in order to arrive at, or close to, the desired vapor fraction. A more detailed description of the experimental procedure is provided in Ref. 22.

\section{RESULTS AND DISCUSSION}

In the following subsections, nucleation rate data will be presented and discussed for water in helium and nitrogen, and for $n$-nonane in helium and methane. For each of these vapor-gas mixtures we present data series at 10, 25, and 40 bar. Throughout this section, a "series" of nucleation experiments is defined as a sequence of wave tube runs with the same nucleation pressure $p_{\text {exp }}$ and temperature $T_{\text {exp }}$, but with a varying vapor fraction or supersaturation. All data are given in tabular form in Ref. 22.

In practice, it is not possible to exactly achieve the desired nucleation temperature, $T_{\text {aim }}$, in each experiment. Fortunately, it is possible to correct for a small scatter in temperature, as was demonstrated by Looijmans. ${ }^{26} \mathrm{He}$ assumed that classical nucleation theory, although not necessarily correct, predicts the right temperature and supersaturation trends for sufficiently small ranges. It is then possible to calculate the shift in $S$ that would be necessary to yield the same nucleation rate, in the case that $T$ would have been exactly equal to $T_{\text {aim }}$. 


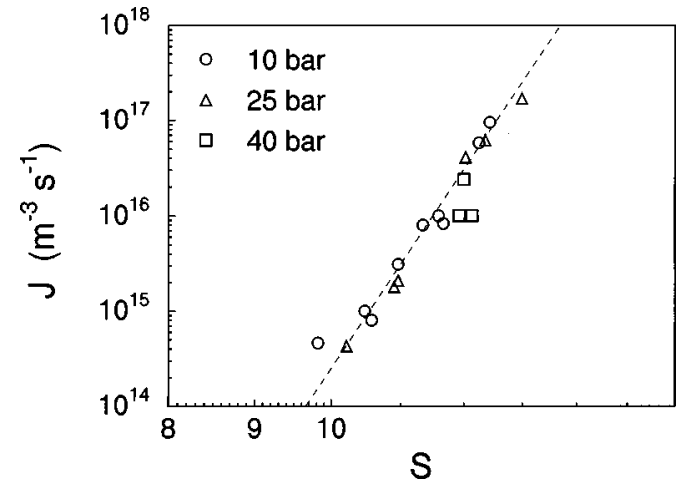

FIG. 2. Experimental nucleation rates for water in helium at $240 \mathrm{~K}$, as a function of (temperature corrected) supersaturation. The dashed line is used to determine the slope of the data series.

Mathematically, we define $S_{\text {corr }}=S+\delta S$, corresponding to $T_{\text {aim }}=T+\delta T$. The correction comes down to evaluating the derivative $(\partial S / \partial T)_{J}$ from classical theory, expressed by Eq. (8) of Paper I. Neglecting the temperature and supersaturation dependencies of the kinetic prefactor, but taking into account the temperature dependence of surface tension, the result is

$$
S_{\text {corr }}=S+\frac{3}{2} \frac{S \ln S}{T}\left[1-\frac{T}{\gamma} \frac{d \gamma}{d T}\right]\left(T-T_{\text {aim }}\right) .
$$

Throughout this section, nucleation data are plotted as a function of this "temperature corrected" supersaturation, allowing for application of the first nucleation theorem, Eq. (13).

Physico-chemical properties of the substances under study, needed in the calculation of theoretical nucleation rates, are summarized in Appendix A of Ref. 22. For the sake of brevity, we will not reproduce the correlations here.

\section{A. Water in helium}

For water-vapor in helium gas, nucleation rates were measured at temperatures near $240 \mathrm{~K}$. The results are plotted as a function of the (temperature corrected) supersaturation in Fig. 2. Using Eq. (13), the number of water molecules in the critical cluster is found from the slope of the isotherms, resulting in $n_{\vee}^{*}=25 \pm 2$ molecules.

Isotherms at different nucleation pressures apparently coincide: Within the experimental scatter, there is no pressure effect on nucleation visible in the water-helium system. Note, however, that the enhancement effect has been taken into account in the calculation of $S$. In this case it is very modest: The saturated vapor density is enhanced with $4 \%$ for a nucleation pressure of 40 bar.

In Fig. 3 the experimental nucleation rates are made dimensionless by their theoretical expectation values at the experimental conditions $(S, T, p)$. All three models are included that were discussed in Paper I: The kinetic classical theory (KCNT), its internally consistent version (ICCT), and the semiphenomenological theory (SPNT) of Kalikmanov and Van Dongen.

Apparently, ICCT gives the closest prediction of experimental nucleation rates. This seems to be a surprising result, in view of the generally spread idea that the original version

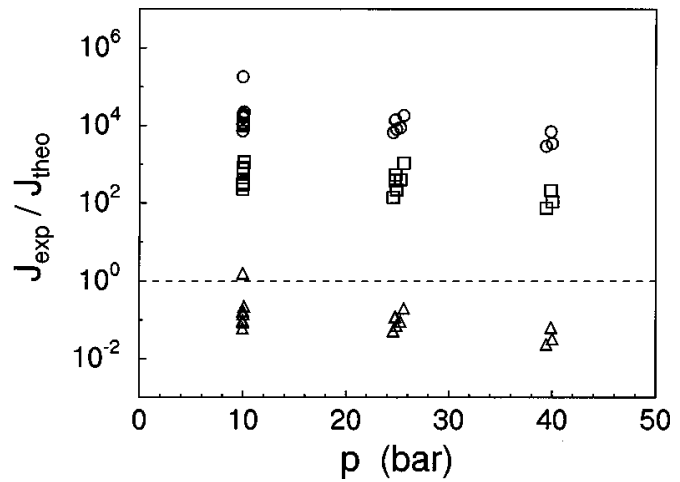

FIG. 3. Ratio of experimental to theoretical nucleation rate for water in helium. Circles: Kinetic classical nucleation theory (KCNT). Triangles: Internally consistent classical theory (ICCT). Squares: Semiphenomenological Kalikmanov-Van Dongen theory (SPNT).

of classical theory performs well for water-vapor. The explanation behind this paradox is that we have used an extrapolation for the surface tension of pure water that differs from the usual correlations. ${ }^{22}$ We used a linear fit, based on experimental surface tension data that were measured down to $-22{ }^{\circ} \mathrm{C}$ by Hacker, ${ }^{27}$ reading

$$
\gamma(T)=0.127245-1.89845 \times 10^{-4} T \quad\left(\mathrm{~N} \mathrm{~m}^{-1}\right) .
$$

The scatter of data points in Fig. 3 that belong to the same series is certainly not caused by measurement noise, as is clear from Fig. 2. It is related to a slight difference in critical cluster size between the experimental result-obtained from the slope of $(J, S)$ isotherms-and model predictions. Values of $n_{\mathrm{v}}^{*}$ and $n_{g}^{*}$ predicted by the pressure perturbation model can be found in Table I.

\section{B. Water in nitrogen}

Nucleation rates for water in nitrogen were also measured at temperatures near $240 \mathrm{~K}$. The resulting data are plotted as a function of the (temperature corrected) supersaturation $S$ in Fig. 4. With help of Eq. (13), we obtain $n_{\mathrm{v}}^{*}$ $=25 \pm 3$.

The slope of the isotherms appears to be the same for all three pressures; yet, they are shifted with respect to each other in vertical direction. According to the "pressure nucleation theorem," Eq. (14), this indicates that nitrogen molecules are present in the critical cluster as well. For an estimate of $n_{g}^{*}$ we neglect the solubility term in Eq. (14), and set the gas compressibility factor equal to unity. The third (enhancement) term is much smaller than unity for the prevailing conditions. From the shift between the 10 and 40 bar isotherms, we obtain $n_{g}^{*} \approx(\partial \log J / \partial \log p)_{s}=3$ for an average pressure of 25 bar. This is very close to the value 2.4 , predicted by the perturbation model of Paper I (see Appendix). Apparently, the latter gives consistent results in terms of critical cluster content.

An appealing question seems to be the "identity" of the carrier gas molecules in the critical cluster: Would it be bulk or surface molecules? According to Eq. (20) of Paper I, the carrier gas content of the bulk liquid core of the cluster is always smaller than the equilibrium solubility. Since the lat- 
TABLE I. Experimental values and model predictions of $n_{v}^{*}$ and $n_{g}^{*}$, as derived from typical values of $\theta$ and $S$ for each series of experiments.

\begin{tabular}{lcccccccc}
\hline \hline System & $p$ (bar) & $T(\mathrm{~K})$ & $\theta$ & $S_{\text {exp }}$ & $n_{\text {v, exp }}^{*}$ & $n_{g, \exp }^{*}$ & $n_{v, \text { model }}^{*}$ & $n_{g, \text { model }}^{*}$ \\
\hline $\mathrm{H}_{2} \mathrm{O}-\mathrm{He}$ & $10-40$ & 240 & 11.6 & 11.5 & 25 & 0 & 32 & 0 \\
$\mathrm{H}_{2} \mathrm{O}-\mathrm{N}_{2}$ & 10 & 240 & 11.5 & 10.0 & 25 & n.a. & 37 & 0.9 \\
$\mathrm{H}_{2} \mathrm{O}-\mathrm{N}_{2}$ & 25 & 240 & 11.4 & 9.3 & 25 & $3^{\mathrm{a}}$ & 40 & 2.4 \\
$\mathrm{H}_{2} \mathrm{O}-\mathrm{N}_{2}$ & 40 & 240 & 11.3 & 8.6 & 25 & n.a. & 43 & 3.9 \\
$\mathrm{C}_{9}-\mathrm{He}$ & $10-40$ & 230 & 18.6 & 100 & 22 & -1 & 20 & 0 \\
$\mathrm{C}_{9}-\mathrm{C}_{1}$ & 10 & 240 & 15.2 & 40 & 26 & n.a. & 21 & 17 \\
$\mathrm{C}_{9}-\mathrm{C}_{1}$ & 25 & 240 & 12.5 & 20 & 18 & $15^{\text {b }}$ & 22 & 39 \\
$\mathrm{C}_{9}-\mathrm{C}_{1}$ & 40 & 240 & 10.3 & 6 & 5 & $22^{\text {c }}$ & 56 & 111 \\
$\mathrm{C}_{9}-\mathrm{C}_{1}$ & 25 & 230 & 21.9 & 20 & 9 & n.a. & 24 & 48 \\
$\mathrm{C}_{9}-\mathrm{C}_{1}$ & 25 & 250 & 12.0 & 20 & 27 & n.a. & 19 & 31 \\
\hline \hline
\end{tabular}

${ }^{\mathrm{a}}$ Pertains to pressure between 10 and 40 bar.

${ }^{\mathrm{b}}$ Pertains to pressure between 10 and 25 bar.

${ }^{c}$ Pertains to pressure between 25 and 40 bar.

ter is very small for nitrogen in water, one must conclude that the carrier gas molecules are located at the surface. On second thought, however, the above question is irrelevant: Regarding the very small total number of molecules in the critical cluster, they can all be regarded of as surface molecules. ${ }^{28}$ We will discuss a remarkable consequence of this feature below.

Figure 5 shows the ratio of experimental to theoretical rates as a function of pressure. Again, the three models are included that were discussed in Paper I (KCNT, ICCT, and SPNT). The surface tension has been obtained from extrapolation of Eq. (7) of Paper I, with a temperature independent value of $p_{L}$. For comparison, calculations with the pure liquid surface tension are shown in Fig. 6. The surface tension effect appears to be able to account for a large part of the observed pressure trend. The remaining slope could still result from extrapolation: The nucleation temperature is $36 \mathrm{~K}$ smaller than the lowest temperature at which $\gamma(p)$ was measured.

Fortunately, the microscopic picture of the critical cluster provides us with independent information. We use the observation made above, that all water molecules can be regarded to reside at the surface; we further assume that each of them may adsorb one nitrogen molecule. We can then derive the surface coverage $\vartheta$, which is an important parameter of the adsorption model in Paper I, from the experimen-

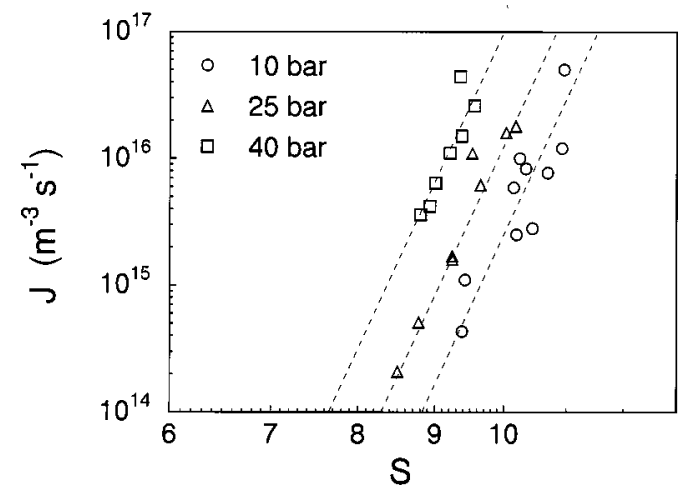

FIG. 4. Experimental nucleation rates for water in nitrogen at $240 \mathrm{~K}$, as a function of (temperature corrected) supersaturation. tally obtained critical cluster composition. With $n_{\mathrm{v}}^{*}=25$ and $n_{g}^{*}=3$, we find $\vartheta=0.12$ for the intermediate pressure of 25 bar. This result can be used to obtain the reference pressure $p_{L}$ from the Langmuir expression (6) of Paper I, yielding $p_{L}=183$ bar.

Figure 7 again shows the ratio of experimental to theoretical nucleation rates, but now with this semi-empirical value of $p_{L}$ used in the surface tension calculation, Eq. (7) of Paper I. Surprisingly, the above value $p_{L}=183$ bar makes the pressure trend vanish altogether. It is tempting to interpret this result as evidence for the adsorption model, although the latter was derived from equilibrium thermodynamics and might have nothing to do with the nucleation problem discussed here. Yet, regardless of the physical reality of the model, the nitrogen adsorption and associated surface tension decrease are able to account in a quantitative sense for the observed dependence of $J$ on $p$.

Figure 8 shows the nucleation results as a function of the reduced partial pressure $y_{\mathrm{v}} p / p^{s}$. The latter corresponds to the "conventional" supersaturation $p_{\mathrm{v}} / p^{s}$; equivalently stated, it is the supersaturation that would exist if there were no enhancement effect. Remarkably, the isotherms belonging to different pressures collapse: For a fixed partial vapor density, the nucleation rate appears to be rather insensitive to total pressure. Two opposing effects are behind this observa-

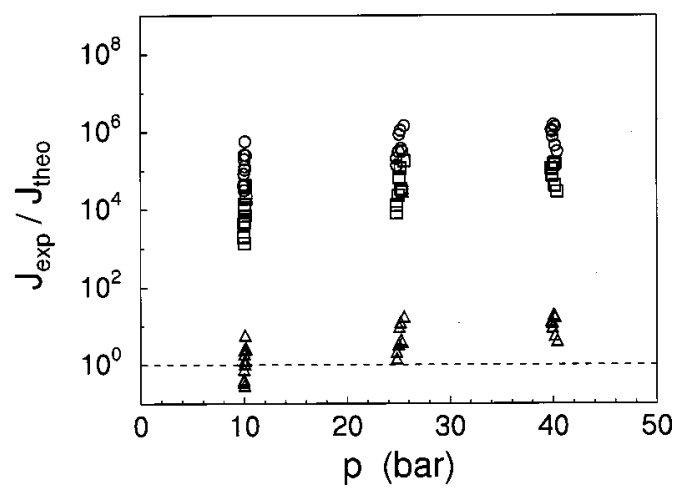

FIG. 5. Ratio of experimental to theoretical nucleation rate for water in nitrogen at $240 \mathrm{~K}$. Circles: KCNT. Triangles: ICCT. Squares: SPNT. 


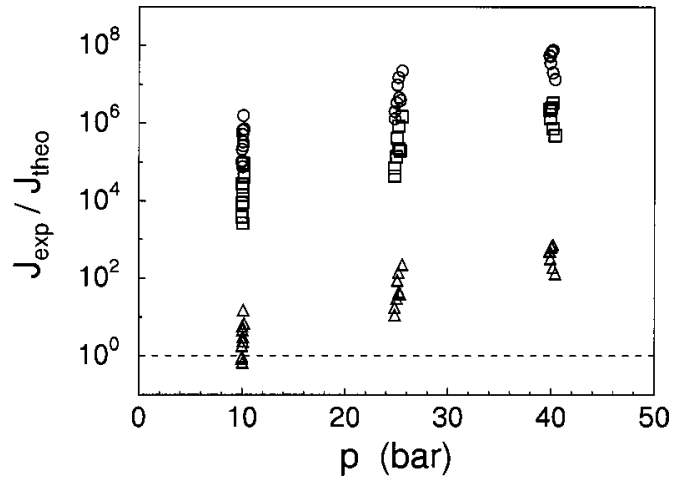

FIG. 6. As Fig. 5, now without inclusion of the pressure dependence of surface tension.

tion. Owing to the enhancement effect, the saturated vapor fraction increases with pressure. At fixed partial vapor density, the supersaturation accordingly decreases, which would cause a decrease of $J$ as well. At the same time, however, the surface tension goes down, causing the nucleation rate to increase. Apparently, these competitive effects are comparable in magnitude under the present conditions.

Since both the enhancement effect and the surface tension decrease are associated with molecular interactions of the condensing vapor with the carrier gas, such a partial compensation can be expected more generally. We used this conjecture in Ref. 10 to explain why pressure trends are seldom reported in nucleation work: If no real gas effects are taken into account in the supersaturation, and the pure liquid surface tension is used, it is very well possible that pressure effects are not observed, despite their occurrence.

\section{C. $n$-Nonane in helium}

Measurements of $n$-nonane nucleation rates in helium gas were performed at temperatures near $230 \mathrm{~K}$. This temperature of $230 \mathrm{~K}$ was imposed by practical conditions: Observing significant nucleation at $240 \mathrm{~K}$ would require higher initial vapor fractions than could be achieved in our setup. Figure 9 presents the results in the familiar $(J, S)$ form. Application of the nucleation theorem (13) results in a critical cluster size of $n_{\mathrm{v}}^{*}=22 \pm 3$ molecules.

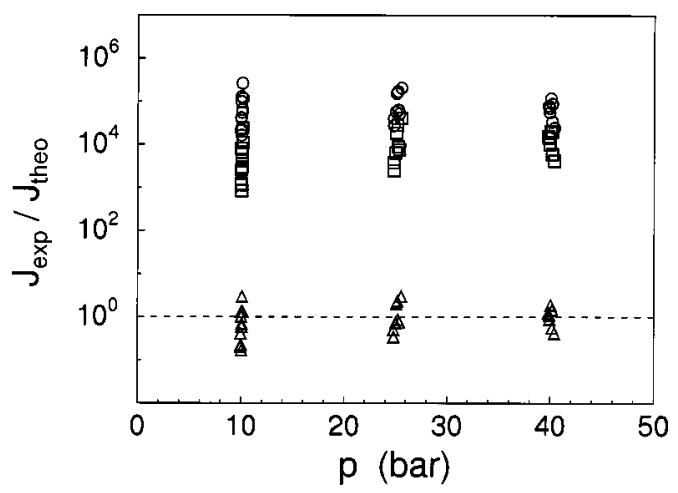

FIG. 7. As Fig. 5, now with Langmuir pressure $p_{L}$ derived from the experimental critical cluster composition.

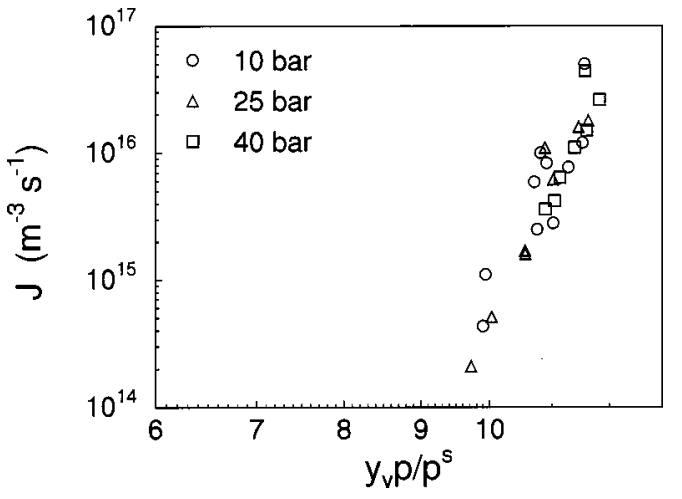

FIG. 8. Experimental nucleation rates for water in nitrogen as a function of the reduced partial pressure $y_{\mathrm{v}} p / p^{s}$.

Just as for water in helium, the three isotherms in Fig. 9 are approximately parallel, indicating that the vapor content of the critical nucleus is insensitive to total pressure. From the vertical distance between the isotherms we find, using Eq. (14), that the carrier gas content would be slightly negative: $n_{g}^{*} \approx-1$.

One of the possible explanations of this result would be that the enhancement effect is underestimated by the Poynting contribution: A very slight positive deviation of $f_{e}$ from its Poynting value would cause the isotherms to collapse, resulting in $n_{g}^{*}=0$. Another possible explanation comes from the gas chromatographic vapor fraction measurement. For the 25 and 40 bar data, vapor fractions were close to the lower limit of the calibration range of the GC setup, resulting in a larger relative uncertainty.

Figure 10 shows the experimental nucleation rates divided by the corresponding theoretical values. One may deduce a small negative trend of $J_{\exp } / J_{\text {theory }}$ with pressure, associated with the arguments above. Both too small values of $f_{e}$ and too large vapor fractions lead to an overestimation of $S$ at higher pressures. Accordingly, the theoretical rate would be too high, which accounts for the negative slope in Fig. 10 . Still, the apparent pressure trend is certainly small enough to exclude any strong influence of pressure in the $n$-nonane-helium mixture at usual nucleation conditions (near atmospheric pressure).

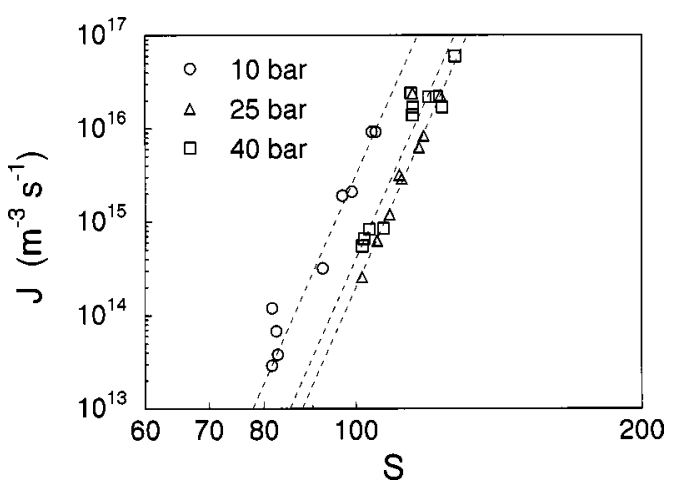

FIG. 9. Experimental nucleation rates for $n$-nonane in helium at $230 \mathrm{~K}$, as a function of (temperature corrected) supersaturation. 


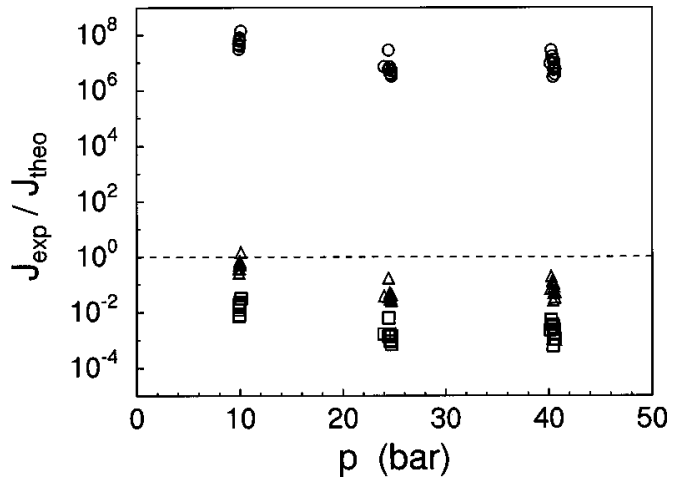

FIG. 10. Ratio of experimental to theoretical nucleation rate for $n$-nonane in helium at $230 \mathrm{~K}$. Circles: KCNT. Triangles: ICCT. Squares: SPNT.

\section{D. $n$-Nonane in methane}

Nucleation rate data in $n$-nonane-methane were again taken at temperatures near $240 \mathrm{~K}$. In addition, the 25 bar measurements were repeated at temperatures of 230 and 250 $\mathrm{K}$. Figure 11 shows the $240 \mathrm{~K}$ results as a function of (temperature corrected) supersaturation $S$.

It is immediately clear that the isotherms for different pressures are no longer parallel, indicating a change in vapor content of the critical cluster with methane pressure. For the 10 and 25 bar series, an accurate determination of the slope in the $(J, S)$ plot is possible. Using Eq. (13) we find $n_{\mathrm{v}}^{*}$ $=26 \pm 1$ at 10 bar and $n_{\mathrm{v}}^{*}=18 \pm 1$ at 25 bar. For the 40 bar data, it is more difficult to deduce a slope. Still, by estimating both upper and lower limiting values, one can deduce that $n_{\mathrm{v}}^{*}=5 \pm 1$.

From the mutual distance between the isotherms, the methane content of the critical cluster can be estimated. In this case the kinetic term in Eq. (14) is not negligible. From Fig. 1 in Paper I we deduce that $\ln f_{e}$ varies from 0.5 to 1 when going from 10 to $25 \mathrm{bar}$, and from 1 to 2 between 25 and 40 bar. Taking this contribution into account, and estimating the vertical distance between isotherms in Fig. 11, we obtain $n_{g}^{*} \approx 15$ between 10 and 25 bar, and $n_{g}^{*} \approx 22$ between 25 and 40 bar. Note that these values of $n_{g}^{*}$ can not be assigned to a unique pressure, since the differential $(\partial \ln J / \partial \ln p)_{S}$ is evaluated from the finite distance between two isotherms that are relatively far apart. ${ }^{29}$

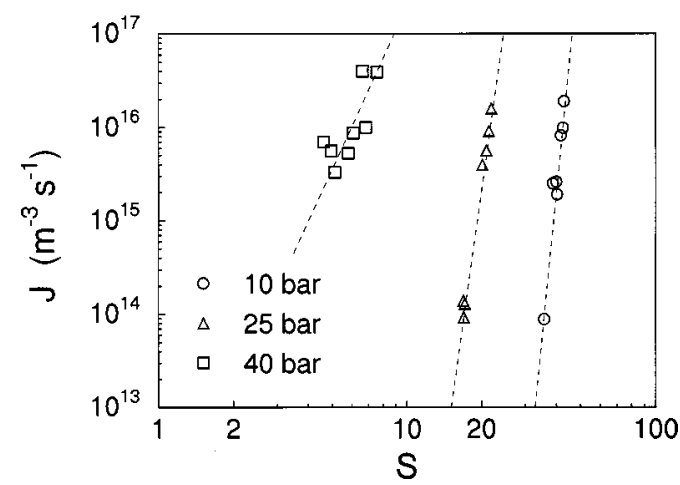

FIG. 11. Experimental nucleation rates for $n$-nonane in methane at $240 \mathrm{~K}$, as a function of (temperature corrected) supersaturation.

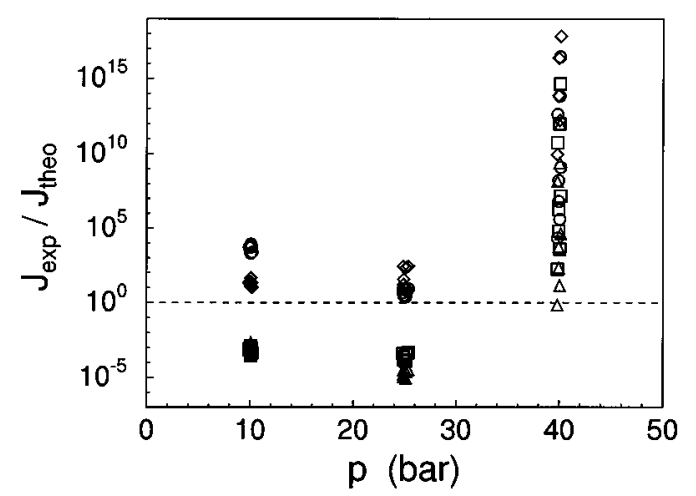

FIG. 12. Ratio of experimental to theoretical nucleation rate for $n$-nonane in methane at 240 K. Circles: KCNT. Triangles: ICCT. Squares: SPNT. Diamonds: BCNT.

The physical picture that emerges from Fig. 11 is the following. With increasing pressure, the supersaturation needed to achieve a certain nucleation rate is dramatically reduced. In other words, higher total pressures greatly facilitate the nucleation process, when its driving force (the supersaturation) is kept fixed. From the application of both nucleation theorems above, it follows that this effect is associated with the presence of more and more methane molecules in the critical cluster-with a molar fraction that is much larger than the equilibrium solubility of methane in liquid $n$-nonane, $x_{g}^{\text {eq }}$. Yet, we have demonstrated in Paper I that the bulk methane fraction must be smaller than $x_{g}^{\text {eq }}$. Hence, the excess amount of methane molecules must be located at the cluster surface. The energy of formation of the $n$-nonane clusters is thereby greatly reduced, leading to an extremely small critical cluster size at the highest nucleation pressure.

The situation is similar to the water-nitrogen case, where adsorption of nitrogen at the cluster surface causes the surface tension to decrease, thus facilitating nucleation. In the present system, however, the effect is much more pronounced. Indeed, the equilibrium surface tension of the mixture decreases very strongly with pressure (see Fig. 3 of Paper I), due to the relatively large methane fraction in the liquid phase.

It is interesting to see how the pressure perturbation approach, presented for the various nucleation models in Paper I of this paper, performs for the present mixture. To that end, experimental nucleation rates are normalized in Fig. 12 by theoretical values of KCNT, ICCT, and SPNT, all including the pressure dependence of the equilibrium surface tension and the enhancement effect. Obviously, the effective onecomponent description is no longer valid. This was to be expected, since the condition underlying the perturbation approach was just small carrier gas solubility in the liquid phase.

A binary model is required to describe the nucleation behavior of the $n$-nonane-methane system. A program code for calculation of nucleation rates using binary classical nucleation theory (BCNT) is available,$^{30}$ which also incorporates the real gas behavior of the mixture. Results for the present data are also included in Fig. 12. For the 10 bar data, the agreement between BCNT and experiments is reason- 


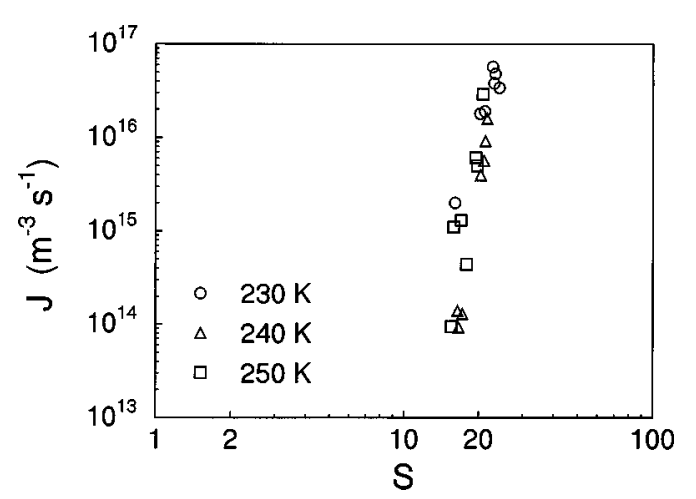

FIG. 13. Nucleation rate as a function of supersaturation for $n$-nonane in methane at 25 bar for nucleation temperatures of 230,240 , and $250 \mathrm{~K}$.

able. At 25 bar the difference gets larger, and for the highest pressure of 40 bar agreement is completely lost.

The main difference between BCNT and the other (onecomponent) models is that the ratio $J_{\exp } / J_{\text {theo }}$ increases with pressure over the whole range, which can be understood on thermodynamic grounds. ${ }^{22}$ Exactly the same observation was reported before by Looijmans et al. ${ }^{11}$ In the latter paper, the vapor fractions were derived from observed droplet growth rates. Fortunately, the results are consistent even in a quantitative sense.

At a total pressure of 40 bar, all theories in Fig. 12including BCNT—clearly fail to predict the experimental nucleation rates within any reasonable limits. The huge scatter is caused by the fact that the critical cluster size (slope of the data series) is not even approximately predicted by any of the models. Since the supersaturations have become very small (Fig. 11), all models predict very low theoretical rates. The equilibrium surface tension is no longer sufficient to explain the magnitude of experimental rates; apparently, the effective surface tension of the cluster is much smaller than its equilibrium value.

In view of the very small experimental value of $n_{\mathrm{v}}^{*}$ at 40 bar, the failure of classical models hardly comes as a surprise: In no way can it be expected that a cluster of five molecules would still have the properties of the macroscopic liquid. It is even quite remarkable that for the other systems and conditions, with critical clusters consisting of 20-30 molecules, classical arguments seem to do such a good job in (qualitatively) explaining the observed nucleation behavior.

As a final illustration of the strong effect of methane pressure on $n$-nonane nucleation, we consider the 25 bar data as a function of nucleation temperature. Figure 13 shows the results in terms of $J$ versus $S$. Quite remarkably, the isotherms for the three different temperatures practically overlap. This could never occur for single component nucleation: With increasing $T$, the isotherms would shift to the left, since both the derivative $(\partial J / \partial T)_{S}$ is positive, and the surface tension generally decreases with increasing temperature. It is the latter argument that forms the key to the explanation of the overlapping isotherms in Fig. 13.

When the temperature is lowered at a fixed total pressure, more methane dissolves into the liquid phase, causing the surface tension to decrease. At certain conditions, this effect can be so strong that the partial derivative $(\partial \sigma / \partial T)_{p}$ becomes positive. For the equilibrium surface tension of the present system this happens at pressures above 40 bar, see Fig. 3 of Paper I. Since the carrier gas fraction in the critical cluster was found to be larger than the equilibrium solubility, however, the effect probably becomes noticeable in the nucleation behavior at a much lower pressure. From Fig. 13 we can conclude that the "reversed surface tension derivative" already plays a role at 25 bar: The surface tension apparently decreases to such extent that the positive derivative $(\partial J / \partial T)_{S}$ is completely balanced.

\section{CONCLUSIONS}

We have presented an extensive series of new nucleation rate data for several vapor-gas systems at pressures of 10 , 25 , and 40 bar. Nucleation of water vapor was studied in the presence of helium and nitrogen gas; $n$-nonane was investigated in carrier gases of helium and methane. Nucleation temperatures ranged from 230 to $250 \mathrm{~K}$.

The experiments were performed in our pulse expansion wave tube setup, with several improvements with respect to earlier work. Vapor fractions of water were measured using an existing humidity sensor, which was now calibrated with the gas pressure as an independent parameter. Hydrocarbon fractions were obtained from gas chromatography, in combination with a thermostatic capillary tube for pressure reduction at constant composition. Both methods were calibrated using a two-bubbler setup, based on controlled saturation of a carrier gas with the vapor under study. The same setup was also used for preparation of the gas-vapor mixtures.

Based on the general formulation of the nucleation theorem for isothermal systems, a relation was derived between the number of carrier gas molecules in the critical cluster and the derivative of the nucleation rate with respect to total pressure. Together with the conventional nucleation theorem for the vapor component, this enables one to derive the full critical cluster composition from experimental $J(S, T, p)$ data.

Both for water and $n$-nonane, helium was found to have no significant influence on the nucleation behavior: $(J, S)$ isotherms at different pressures nearly coincide, indicating that the number of vapor molecules in the critical cluster is not affected by helium pressure, and that helium molecules do not make part of the critical cluster.

For water in nitrogen, $(J, S)$ isotherms at different pressures are still parallel, which shows that the number of vapor molecules in the critical cluster is independent of nitrogen pressure. However, the isotherms are shifted with respect to eachother in the vertical direction, which indicates the presence of nitrogen molecules in the critical cluster. Deriving the number of nitrogen molecules from the pressure nucleation theorem, we were able to compute the surface adsorption and the associated decrease of surface tension with pressure. Substituting this result in the theoretical models of Paper I, we were able to fully account for the observed pressure dependence of the nucleation rate.

None of the theoretical models was able to account for the observed nucleation behavior of $n$-nonane in methane. The breakdown of all theoretical approaches in the latter system can be ascribed to enhanced dissolution of methane into the liquid phase. The methane fraction in the critical cluster 
was found experimentally to be much larger than the equilibrium solubility, leading to a severe decrease of the energy of formation. Hence, fewer $n$-nonane molecules are needed to form a stable cluster, which explains the observed small number of $n$-nonane molecules in the critical nucleus.

Summarizing the results of Papers I and II, the most important conclusion might be that the high pressure features of the phase equilibrium of a binary mixture are recovered in the nucleation behavior. This concerns mainly the two effects that have been extensively discussed in Paper I, being the increase of saturated vapor content with pressure (enhancement effect) and the accompanying decrease of surface tension.

Accounting for these two effects, a description of the new nucleation results at high pressure could be given in terms of the pressure perturbation approach proposed in $\mathrm{Pa}$ per I. Only for the $n$-nonane-methane mixture, this approach was not able to account for the observed nucleation behavior. This corresponds to the validity criterion derived in Paper I: In order for the pressure perturbation approach to be valid, the carrier solubility has to be sufficiently small.

\section{APPENDIX CRITICAL CLUSTER CONTENT DERIVED FROM PRESSURE PERTURBATION MODEL}

The nucleation rate according to the pressure perturbation model of Paper I can be written in the familiar form

$$
J=K \exp \left[-\frac{4}{27} \frac{\theta^{3}}{(\ln S)^{2}}\right],
$$

the essential difference with one-component theory being that the dimensionless surface tension $\theta$ now depends on pressure. On differentiating (A1) we obtain

$$
\left[\frac{\partial \ln (J / K)}{\partial \ln S}\right]_{p, T}=\left(\frac{2 \theta}{3 \ln S}\right)^{3} .
$$

Since it is only the exponential factor in Eq. (A1) that is related to the critical numbers, we consider the derivative of $J / K$. According to Eq. (13) the right hand side is equal to $n_{\mathrm{v}}^{*}$. The pressure derivative is

$$
\left[\frac{\partial \ln (J / K)}{\partial \ln p}\right]_{S, T}=-\frac{12}{27}\left(\frac{\theta}{\ln S}\right)^{2} \frac{\partial \theta}{\partial \ln p} .
$$

Using the adsorption model described in Paper I, the derivative $\partial \theta / \partial \ln p$ can be evaluated, resulting in

$$
\left[\frac{\partial \ln (J / K)}{\partial \ln p}\right]_{S, T}=\frac{12}{27}\left(\frac{\theta}{\ln S}\right)^{2} \frac{n_{a} a_{0} p}{p+p_{L}} .
$$

According to Eq. (14) the right hand side now equals $-n_{\mathrm{v}}^{*} x_{g}^{\mathrm{eq}}+n_{g}^{*} Z_{g}$. Combining the results for $n_{\mathrm{v}}^{*}$ and $n_{g}^{*}$ we obtain

$$
n_{g}^{*}=\frac{1}{Z_{g}}\left[\frac{12}{27}\left(\frac{\theta}{\ln S}\right)^{2} \frac{n_{a} a_{0} p}{p+p_{L}}+x_{g}^{\mathrm{eq}}\left(\frac{2 \theta}{3 \ln S}\right)^{3}\right] .
$$

Using values for $p_{L}$ from part I and literature data for $Z_{g}$ and $x_{g}^{\mathrm{eq}}$, the model predictions of $n_{g}^{*}$ can be evaluated. The results are summarized in Table I. Since the surface tension of water and $n$-nonane is not lowered by helium pressure, the $p_{L}$ values for both helium systems were set to infinity (corresponding to zero adsorption).

${ }^{1}$ R. H. Heist and H. He, J. Phys. Chem. Ref. Data 23, 781 (1994).

${ }^{2}$ P. van Remoortere, C. Heath, P. E. Wagner, and R. Strey, in Nucleation and Atmospheric Aerosols, edited by M. Kulmala and P. E. Wagner (Pergamon, Oxford, 1996), p. 256.

${ }^{3}$ J. A. Fisk and J. L. Katz, J. Chem. Phys. 104, 8649 (1996).

${ }^{4}$ R. H. Heist, M. Janjua, and J. Ahmed, J. Phys. Chem. 98, 4443 (1994).

${ }^{5}$ D. Kane and M. S. El-Shall, J. Chem. Phys. 105, 7617 (1996).

${ }^{6}$ J. A. Fisk, V. M. Chakarov, and J. L. Katz, J. Chem. Phys. 104, 8657 (1996).

${ }^{7}$ A. Bertelsmann and R. H. Heist, J. Chem. Phys. 106, 610 (1997).

${ }^{8}$ A. Bertelsmann and R. H. Heist, J. Chem. Phys. 106, 624 (1997).

${ }^{9}$ M. J. E. H. Muitjens, Ph.D. thesis, Eindhoven University of Technology, 1996.

${ }^{10}$ C. C. M. Luijten, K. J. Bosschaart, and M. E. H. van Dongen, J. Chem. Phys. 106, 8116 (1997).

${ }^{11}$ K. N. H. Looijmans, C. C. M. Luijten, and M. E. H. van Dongen, J. Chem. Phys. 103, 1714 (1995)

${ }^{12}$ K. N. H. Looijmans, P. C. Kriesels, and M. E. H. van Dongen, Exp. Fluids 15, 61 (1993).

${ }^{13}$ K. N. H. Looijmans and M. E. H. van Dongen, Exp. Fluids 23, 54 (1997).

${ }^{14}$ C. C. M. Luijten and M. E. H. van Dongen, J. Chem. Phys. 111, 8524 (1999), preceding paper.

${ }^{15}$ D. Kashchiev, J. Chem. Phys. 76, 5098 (1982).

${ }^{16}$ D. W. Oxtoby and D. Kashchiev, J. Chem. Phys. 100, 7665 (1994).

${ }^{17}$ I. J. Ford, J. Chem. Phys. 105, 8324 (1996).

${ }^{18}$ I. J. Ford, Phys. Rev. E 56, 5615 (1997).

${ }^{19}$ D. W. Oxtoby and A. Laaksonen, J. Chem. Phys. 102, 6846 (1995)

${ }^{20}$ J. L. Katz, J. A. Fisk, and V. Chakarov, in Nucleation and Atmospheric Aerosols, edited by N. Fukuta and P. E. Wagner (Deepak, Hampton, 1992), p. 11

${ }^{21}$ R. C. Reid, J. M. Prausnitz, and B. E. Poling, The Properties of Gases and Liquids (McGraw-Hill, New York, 1987).

${ }^{22}$ C. C. M. Luijten, Ph.D. thesis, Eindhoven University of Technology, ISBN 90-386-0747-4, 1998.

${ }^{23}$ C. C. M. Luijten, L. E. Stormbom, and M. E. H. van Dongen, Sens. Actuators B 49, 279 (1998)

${ }^{24}$ R. G. Wylie and R. S. Fisher, Chem. Eng. Data Ser. 41, 133 (1996).

${ }^{25}$ C.-H. Hung, M. J. Krasnopoler, and J. L. Katz, J. Chem. Phys. 90, 1856 (1989).

${ }^{26}$ K. N. H. Looijmans, Ph.D. thesis, Eindhoven University of Technology, 1998.

${ }^{27}$ P. T. Hacker, National Advisory Committee for Aeronautics, technical note $2510,1951$.

${ }^{28}$ Consider, for example, the imaginary case of a cubic cluster with cubic molecules: for $n_{\mathrm{V}}^{*}+n_{g}^{*}=27$, only one of them is in the centre and could be considered as a "bulk" molecule.

${ }^{29}$ In order to arrive at more accurate $n_{g}^{*}$ values, one would have to increase the nucleation pressure in small steps, keeping the saturation ratio constant. In practice, such a procedure is difficult to realize.

${ }^{30}$ K. N. H. Looijmans, C. C. M. Luijten, G. C. J. Hofmans, and M. E. H. van Dongen, J. Chem. Phys. 102, 4531 (1995). 